\title{
Gestational Trophoblastic Tumor
}

National Cancer Institute

\section{Source}

National Cancer Institute. Gestational Trophoblastic Tumor. NCI Thesaurus. Code C4699.

A diverse group of pregnancy-related tumors characterized by excessive proliferation of trophoblasts. Representative examples include hydatidiform mole, gestational choriocarcinoma, and placental site trophoblastic tumor. 